\title{
Novel quantum NMR magnetometer non-contact defectoscopy and monitoring technique for the safe exploitation of gas pipelines
}

\author{
E. D. Narkhov, V. A. Sapunov, A. U. Denisov \& D. V. Savelyev \\ Ural Federal University named after the first President of Russia \\ B. N. Yeltsin, Russian Federation
}

\begin{abstract}
Pipeline transportation has already proved to be a high-performance tool of resources transportation for the efficient functioning of modern society. However, an aggressive environment, the modernization of existing pipelines and the building of new pipelines pose a number of problems to be solved for secure exploitation. Such problems include mapping, systems certification, technical inspection and monitoring. Recently much attention has being paid to the effective solution of these problems, with no interference into the functioning of the existing systems (non-contact methods), and the magnetometric technique is one such method. The method presented in this paper is based on the interpretation of the absolute value of the magnetic field of an object, which allows us to carry out measurements more accurately compared to other approaches. This paper presents the preliminary results of the usage of high-precision absolute quantum Overhauser "POS" (proton Overhauser sensor) magnetometers in the oil-and-gas field. The field work conducted in the summer of 2013 showed that this equipment has great potential for safe exploitation of oil-and-gas pipelines. The efficiency of the geophysical equipment for gas pipelines of a large diameter (1400 mm) was also confirmed under actual operating conditions.

Keywords: magnetometer, pipeline, non-contact defectoscopy, non-destructive control.
\end{abstract}




\section{Introduction}

The active usage of pipeline transport has given rise to practical tasks aimed at the safe and reliable exploitation of pipeline systems, including their mapping, certification, defectoscopy and monitoring. The safe operation of the pipelines is complicated by many factors such as: length, variety of bedding environment, and severe conditions of exploitation. Technological developments, based on the use of modern equipment, would improve the safety of pipeline exploitation.

This paper presents the results of the non-contact magnetometric method using absolute quantum nuclear magnetic resonance (NMR) Overhauser magnetometers. By the principle of operation they enter a class of proton magnetometers whose measurements are based on the relation of the free precession frequency $\omega$ of the substance nuclei to the modulus of induction of the external magnetic field $|\vec{B}|_{0}$ :

$$
\omega=\gamma\left|\vec{B}_{0}\right|
$$

The knowledge of the gyromagnetic ratio of proton $\gamma$ with high precision (up to $10^{-8}$ ) provides high measurement accuracy. This method has great potential for the development of non-contact control methods improving pipeline safety.

\section{Survey methodology of gas pipeline}

\subsection{Magnetic field of the pipeline}

The practical application of a proton magnetometer for the examination of pipelines was mentioned in one of the first publications in this field [1], dealing with the detection of fatigue stresses in a pipeline. It most clearly formulated the main provisions of the survey methodology of pipelines: 'Pipelines constructed on unstable terrain (e.g. permafrost), submarine lines, etc. may shift, and producing stresses which can lead ultimately to rupture. We have used magnetometer surveys to detect bending stresses in buried pipelines and to monitor new construction. The perturbations to the earth's field are large and complex; typically fluctuations of 5-10 kilogamma are found $2 \mathrm{~m}$ above $1 \mathrm{~m}$ diameter buried line. These signals record initial magnetizations, joints, construction history, etc. and include the results of stress induced magnetization changes.' The method under consideration is based on the relation between deformation processes (often accompanied by defects formation) and magnetometric anomalies [2].

We should distinguish the application of proton magnetometers which find the location [3] and defects $[4,5]$ of joint welds that might be useful for defectoscopy, mapping and pipeline certification. The actual magnetic profile of a pipeline is rather complicated as it includes:

- magnetically hard and soft magnetization of pipes themselves;

- field of joint welds with their defects [3, 4]; 
- anomalies related with the stresses appeared during the process of installation, as well as due to the natural dynamic processes in the base of pipelines [1];

- $\quad$ anomalies due to defects $[1,5]$;

- the magnetic field of anthropogenic objects located near the pipeline, as well as the heterogeneity of the pipeline installation [6].

For an efficient solution to the problems related to pipeline magnetometry it is important to make a correct choice of a physical model of the object under consideration according to which the anomalies of the magnetic field would be registered. Thus, it is possible to mark defects and classify the level of their criticality according to the form and amplitude of anomalies.

In the simplest case, the magnetic field of a hard-magnetic component of a separate pipe can be represented as a field of bar magnet $[1,3]$. According to this approach, the model of a pipeline is represented as randomly oriented dipole magnets or current rings distributed alongside the straight line. The existing experimental data showed that this model does not fit well to the practical tasks. In this paper we use a more detailed approach using a system of thin-walled solenoids as a pipeline model.

\subsection{Theoretical model of the pipeline magnetic field}

A hard magnetic field component of a magnetized cylinder can be modeled by the field of an infinitely thin solenoid located on the cylinder surface with a surface density of a current equal to the magnetization of the cylinder [7]. The general expression for the field of thin-walled solenoid is rather bulky and can be found in reference books, for example [8]. Thus, the field of the pipe is presented as a sum of fields from two coaxial oppositely-connected infinitely thin solenoids located along the inner and outer wall of a pipe with a current density equal to the pipe magnetization. We have chosen the value of magnetization equal to $70 \%$ of the specific saturation magnetization [6] for the pipe material.

To highlight the anomalies, a computer modeling of the magnetic survey was carried out at different configurations of pipelines (various combinations of magnetization direction of pipes, pipe dimensions) and measurement conditions (configuration of sensors, mesh spacing, etc.).

It was taken into account that the construction of a map of the pipe magnetic field was carried out under conditions of an external magnetic field at different mutual orientations (see Figure 1, Figure 2).

It should be noted that the value of the Earth field $B_{0}$ is almost always much larger than the magnitude of the field of pipeline $B_{p}$, which allows us to use the following approximation:

$$
\left|\vec{B}_{0}+\vec{B}_{p}\right|=B_{0}+B_{p} \cos \varphi
$$

where $\varphi$ is the angle between field directions. 


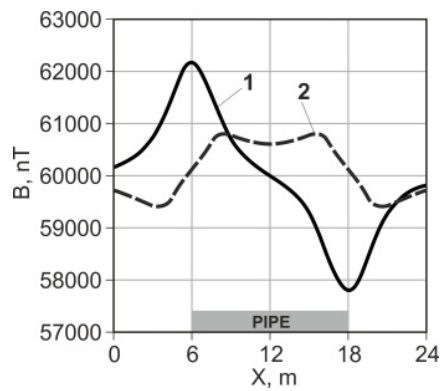

Figure 1: Theoretical profiles of the pipe magnetic field constructed above the pipe axis when the orientation of the external field is: 1 . perpendicular to the pipe axis in the vertical plane; 2. along the pipe axis.

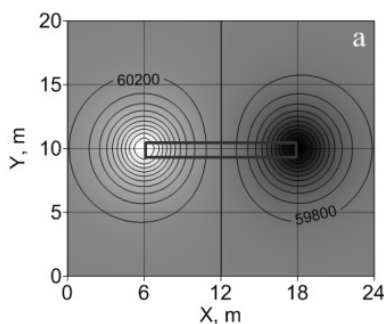

(a)

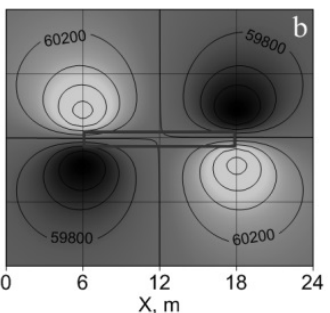

(b)

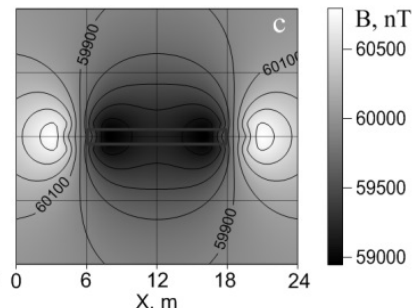

(c)

Figure 2: Magnetic maps of the pipe field at different orientations of the external field: (a) perpendicular to the tube axis in the vertical plane; (b) perpendicular to the tube axis in the horizontal plane; (c) along the pipe axis.

The resultant pipeline field is modeled by a superposition of the solenoids, where the direction of magnetization conforms to the most adequate reproduction of the real pipeline measurements. This allows us to mark out the anomalies related to potential defects.

\section{Magnetometer surveys of gas pipelines}

\subsection{Quantum NMR proton Overhauser magnetometers - gradiometer}

Quantum NMR proton Overhauser magnetometers are well-known; they are widely used in ground, marine, and borehole geological prospecting. The reason for such proliferation, as compared with other known magnetometers (like flux-gate, GMR, Hall), is the measurement of the absolute value (length of the field vector), based on the physical principles of sensors [9]. The absolute scalar sensor does not require orientation in space and thus has no angular errors. Certainly, valuable information on the direction of the anomalous field is lost, but 
the highest accuracy of up to $0.0001 \%$ is provided, which enhances interpretation. The fundamental measurement formula (1) is presented above. Table 1 shows the diagram of the proton and Overhauser magnetometer.

Table 1: Diagram of the proton and Overhauser magnetometer.

\begin{tabular}{|c|c|c|}
\hline Scheme & Time diagram & Notes \\
\hline Liquid & $\mathrm{M}_{\mathrm{z}}$ & $\begin{array}{c}\text { Packard-Varian method [10] } \\
\mathrm{B}_{\mathrm{pol}} \approx 100 \div 300 \mathrm{Oe}\end{array}$ \\
\hline
\end{tabular}

At the first stage the nuclei of the working substance (liquid) is magnetized by the polarizing field, and then the field is quickly turned off. At the second stage the nuclei freely precess as spintops. This type of magnetometer is primarily used in work dedicated to the magnetometer surveys of pipelines [1]. Such magnetometers have a limited useful signal, which makes it difficult to work with strong magnetic objects, such as pipes. The Overhauser effect results in a tremendous increase of the signal and decrease of the energy consumption. It is necessary to use a liquid with the addition of special radicals, which are carriers of electron paramagnetic resonance, which increases the saturation of the nuclear signal due to the presence of quantum interactions at the nanolevel. The research laboratory of quantum magnetometry at UFU is one of the leaders in the design of such substances and magnetometer production. These magnetometers were used in this research.

The measurements were carried out using two standard quantum geological magnetometers: «MMPOS-1gps» and gradiometer «MMPOS-2gps». The magnetometer "POS-1" and gradiometer "POS-2" are registered in the State Register of Measuring in Russian Federation № 44807-10, with an approval certificate of the measuring type RU.C.34.005.A № 40335. TU 4314-001020692208-2007.

On the one hand the metrological parameters of these magnetometers exceed the required level for detecting anomalies in pipelines by magnetometer surveys [12]. On the other hand, precision measurements allow for the consideration of the long-term monitoring of pipelines. 
One of the critical parameters of the proton magnetometers' applicability for strong-magnetic objects is the so-called gradient tolerance. Standard proton magnetometers are often unable to work under these conditions. The Overhauser magnetometers, which use special algorithms implemented in POSmagnetometers, considerably improve the situation [13]. According to calculations the magnitude of the magnetic field gradients should reach values of up to $30000 \mathrm{nT} / \mathrm{m}$ (for a pipeline with a diameter of up to $1400 \mathrm{~mm}$ ). The research of the gradient tolerance parameter of a weak magnetic field, under standard laboratory conditions, is carried out for the selected equipment according to the method [14]. The value of gradient tolerance obtained reaches $40,000 \mathrm{nT} / \mathrm{m}$, which meets the requirements of the work at the pipelines.

\subsection{General information about the measurements conducted}

The magnetic prospecting on gas pipelines of a large diameter was conducted in the summer of 2013. Measurements were carried out at pipelines with a diameter of $1400 \mathrm{~mm}$ and $1200 \mathrm{~mm}$ in the Sverdlovsk region. Some of them were in exploitation and the others were under major repair. The pipelines explored consisted of both single-joint and double-joint pipes, which have been tested using the method of in-line inspection (pigging) during the last 5 years.

During work on the first object, the general availability of the selected equipment was tested in different modes (system configuration, measurement period, geographical reference). Work on the second object was held in three stages. During the first stage the measurements were performed by two magnetometer operators (Figure 3, left). Then the magnetometer configuration and methods of measurements were modified. During the second stage the measurements were performed by a single operator with the prototype of multisensors systems (Figure 3, right). The third stage was dedicated to the measurement of magnetic fields at the defective sections of pipelines. The manuscript presents the results of the second stage measurements.
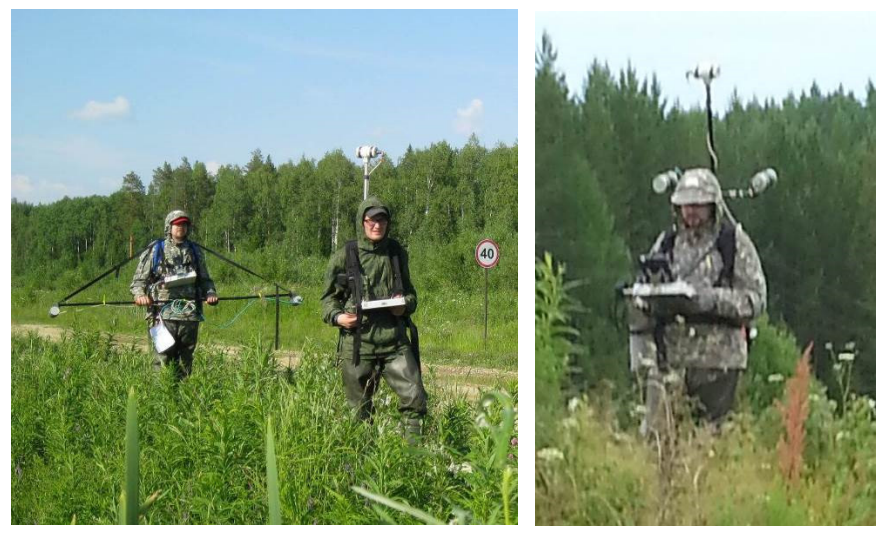

Figure 3: Magnetic diagnostic of gas pipeline by two operators (left) and by prototype of multi-sensors magnetometer-gradientometer (right). 


\subsection{Data of magnetometric measurements}

According to the results of field work, the magnetometric data concerning the magnetic profile of the two kilometers long gas pipeline for both forward and reverse motion was obtained. The data for a segment of the pipeline between markers $-560 \mathrm{~m}-0 \mathrm{~m}$ is shown in Figure 4, including the profile of quality measurement criterion (QMC) (Figure 4a), the transverse gradient of the magnetic field induction module above the axis of the pipeline (Figure 4b), the profile of the magnetic field above the axis of the pipeline (Figure 4c) and the markers of the potential defects are presented on the left side of the Figure. The QMC parameter estimates of the instrumental accuracy for each measurement also related to the gradient in the sensor volume [14].

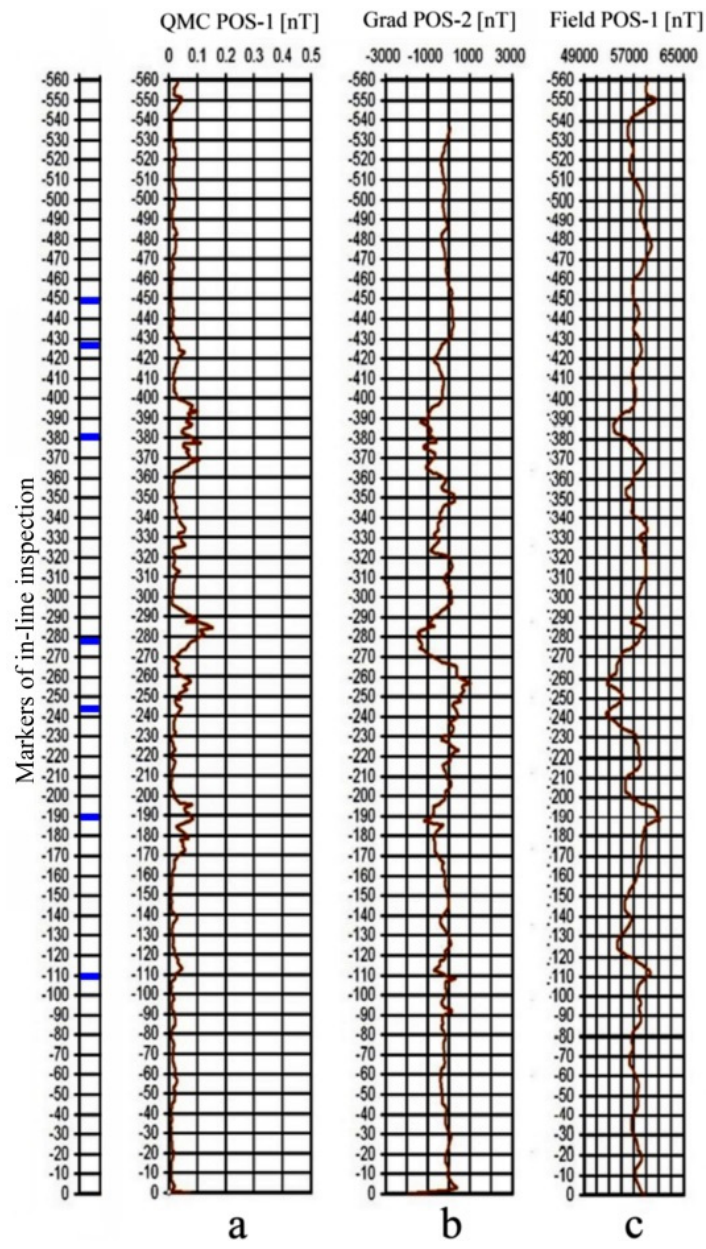

Figure 4: Magnetometric data of non-contact magnetometric inspection for section of the pipeline. 


\subsection{Data analysis}

We used the theoretical model described above for the data analysis. Potential zones of the existing or emerging defects were determined according to $\mathrm{RD}$ (guidance document) 102-008-2002 [16]. These zones are characterized by a high level of deformation stresses that rather clearly stand out on the vertical and lateral gradients. A comparison of the magnetic data with ILI-data showed a high correlation. The third stage was performed after marked defects digging. The measurements of the magnetic field over the pits showed that the shape and amplitude anomalies of the defects could potentially allow the classification of defects (corrosion, weld defect, cracking of pipe body, dents) using the non-contact method. The amplitude anomaly characterizes the degree of development/criticality of the defect.

The improvement of the method's efficiency is associated with the exclusion of welding noise (anomalies associated with welds and chaotic magnetization direction of individual tubes). This follows from theoretical and computer simulations, combined with the modern methods of geological exploration magnetometry. The existing model allows us to perform the analysis by taking into account a sharp deviation of the planar gradient from zero value. It also accounts for the value of the observed gradients, gradient-change frequencies, and the relative lengths of pipes making up the body of pipelines. Additionally, the data's reproducibility analysis was carried out. The magnetic profile for forward and reverse motion were measured and compared to each other. The measured sections are similar at $86-99 \%$ level. The increase of reproducibility is possible due to the use of high-precision GPS equipment. Increased reproducibility represents an advantage in comparison with the standard equipment.

\section{Conclusions and discussion}

The functionality of standard geological absolute quantum Overhauser magnetometers and gradiometers «MMPOS» is shown during this research under the field conditions. The measurement of pipelines of different diameters, made of different types of pipes, was performed. The high reproducibility of measurements is demonstrated, thus leading to a new technical control type - a monitoring survey. This type of technical control cannot only find the existing defects, but can also track the formation of new defects at the early stages.

Some requirements for the modernization of the existing system are formulated. Specialization of standard magnetometer «MMPOS» by increasing the speed of response (with decreasing sensitivity) would improve efficiency.

We believe that the usage of multi-sensors systems would not only be promising, but would also allow us to extend the number of problems solved with the help of this system.

The directions of the theoretical model development are formulated. The accumulation of a magnetic database for the analysis of various defects and the development of non-contact type of defect classification by the magnetometric survey is required. The comparison of obtained data with the ILI is performed, 
showing good correlation. The level of correlation will improve by increasing the quality of GPS positioning.

The further development of the non-contact magnetometric technique would allow us to improve the detection of pipeline defects. Thus the number of accidents and environmental disasters related to the destruction of pipeline systems will be reduced.

\section{References}

[1] Atherton, D. L., Teitsma, A., Detection of anomalous stresses in gas pipelines by magnetometer survey (invited). Journal of Applied Physics, 53(11), pp. 8130-8135, 1982.

[2] Dubov, A., Kolokolnikov, S., Assessment of the material state of oil and gas pipelines based on the metal magnetic memory method. Welding in the World, 56(3-4), pp. 11-19, 2012.

[3] Nekrasov, I.A., Denisov, A. Yu., Kiselev, S.E., Savel'ev, D.V., Sapunov, V.A., A proton magnetometer used to locate pipeline welds. Russian Journal of Nondestructive Testing, 34(3), pp. 190-194, 1998.

[4] Novikov, V.A., Romanov, V.A., Magnetic control of local defects in welded joints. Defektoskopiya, 1, pp. 32-39, 1994.

[5] Reutov, Yu. Ya., Loskutov, V.E., Gobov, Yu. L., Vaulin, S.L., Magnetic field of a circular butt weld of a trunk oil and gas pipeline. Russian Journal of Nondestructive Testing, 39(11), pp. 852-860, 2003.

[6] Krapivsky E.I., Nekuchaev V.O., Remote control of gas and oil pipelines. Published by Ukhtinsky State Technical University, 2011 (in Russian).

[7] Purcell, Edward M., Electricity and Magnetism (In SI Units): Berkeley Physics Course, Volume 2. Published by Tata McGraw-Hill Education Pvt. Ltd., 2011.

[8] Sargsyan L. A., Analytical methods for calculating the stationary magnetic fields: reference manual. Published by Energopromizdat, Moscow, 1993.

[9] Ripka, Pavel, Magnetic sensors and magnetometer. Published by Artech House, 2001.

[10] Packard, M.E., Varian, R.H., Free nuclear induction in the Earth's magnetic field. Physical Review, 93, p. 941, 1954.

[11] Shifrin, V. Ya., Khorev, V. N. , Kalabin, V. N. , Park, P. G., Experimental estimation of the accuracy of modern scalar quantum magnetometers in measurements of the Earth's magnetic field. Physics of the Earth and Planetary Interiors, 166(3-4), pp. 147-152, 2008.

[12] Sapunov, V., Denisov, A., Denisova, O., Saveliev, D., Proton and Overhauser magnetometers metrology. Contributions to Geophysics and Geodesy, 31(1), pp. 119-124, 2001.

[13] Denisova, O., Sapunov, V., Denisov, A., Influence of magnetic field variations on measurements by magnetometers using averaging algorithms. Earth, Planets and Space, 58(6), pp. 731-734, 2006.

[14] Sapunov, V.A., Savelyev, D.V., Denisov, A.Y., Kiselev, S.E., Saveliev, V.D., Narhov, E.D., TianBiao W., XiaoShen H., XiangDong C., Gradient 
Tolerance Measurement Method of LOM-2 Borehole Overhauser Magnetometer in Laboratory Conditions. China International Symposium on Geophysical Instruments and their application. The fifth conference of geophysical technology. Guide and abstracts. Elsevier: Beijing, pp. 54-63, 2012.

[15] Denisov, A.Y., Denisova, O.V., Sapunov, V.A., Khomutov, S.Y., Measurement quality estimation of proton-precession magnetometers. Earth, Planets and Space, 58(6), pp. 707-710, 2006.

[16] RD 102-008-2002 "Instructions for diagnosing the technical condition of the pipelines by non-contact magnetometric method" (management directive). Publication of the Russian State Technical Supervision, 2002. http://minenergo.gov.ru/documents/normative/8703.html 